\section{OPEN ACCESS}

Edited and reviewed by: Cirino Botta

Cosenza Hospital, Italy

${ }^{*}$ Correspondence: Qingxin Xia tudou414135404@163.com Mingzhi Zhang mingzhi_zhang1@163.com

${ }^{\dagger}$ These authors have contributed equally to this work

Specialty section:

This article was submitted to

Cancer Molecular Targets and Therapeutics,

a section of the journal

Frontiers in Oncology

Received: 15 July 2020 Accepted: 29 September 2020 Published: 27 January 2021

Citation:

Song $Y$, Song $W$, $L i Z$, Song $W$, Wen Y, Li J, Xia Q and Zhang M (2021)

Corrigendum: CDC27 Promotes

Tumor Progression and Affects

PD-L1 Expression in T-Cell

Lymphoblastic Lymphoma.

Front. Oncol. 10:583698.

doi: 10.3389/fonc.2020.583698

\title{
Corrigendum: CDC27 Promotes Tumor Progression and Affects PD-L1 Expression in T-Cell Lymphoblastic Lymphoma
}

\begin{abstract}
Yue Song ${ }^{1,2,3+}$, Wei Song ${ }^{4 \dagger}$, Zhaoming $\mathrm{Li}^{1,3}$, Wenting Song ${ }^{1,2,3}$, Yibo Wen ${ }^{2}$, Jiwei $\mathrm{Li}^{1,3}$, Qingxin $\mathrm{Xia}^{4 *}$ and Mingzhi Zhang ${ }^{1,3 *}$

1 Department of Oncology, The First Affiliated Hospital of Zhengzhou University, Zhengzhou, China, 2 The Academy of Medical Science of Zhengzhou University, Zhengzhou, China, ${ }^{3}$ Lymphoma Diagnosis and Treatment Center of Henan Province, Zhengzhou, China, ${ }^{4}$ Department of Pathology, The Affiliated Cancer Hospital of Zhengzhou University, Henan Cancer Hospital, Zhengzhou, China
\end{abstract}

Keywords: CDC27, T-cell lymphoblastic lymphoma, PD-L1, cell cycle, APC/C

\section{A Corrigendum on}

CDC27 Promotes Tumor Progression and Affects PD-L1 Expression in T-Cell Lymphoblastic Lymphoma

Song Y, Song W, Li Z, Song W, Wen Y, Li J, Xia Q and Zhang M (2020). Front. Oncol. 10:488. doi: 10.3389/fonc.2020.00488

In the original article, there was a mistake in Figure $\mathbf{3}$ and Figure $\mathbf{4}$ as published. In Figure $\mathbf{3}$, we put the wrong picture of EdU result in shNC group. In Figure 4B, we put the wrong picture of Apoptosis result in Control group. The correct Figure $\mathbf{3}$ and Figure $\mathbf{4}$ appear below. And in the Materials and Methods parts, we give a supplementary statement that cell lines used for Immunofluorescence were transfected with plasmid without GFP tag.

The authors apologize for these errors and state that this does not change the scientific conclusions of the article in any way. The original article has been updated.

Copyright (C) 2021 Song, Song, Li, Song, Wen, Li, Xia and Zhang. This is an open-access article distributed under the terms of the Creative Commons Attribution License (CC BY). The use, distribution or reproduction in other forums is permitted, provided the original author(s) and the copyright owner(s) are credited and that the original publication in this journal is cited, in accordance with accepted academic practice. No use, distribution or reproduction is permitted which does not comply with these terms. 
A

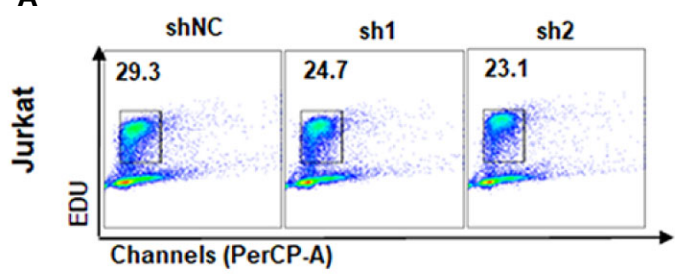

B

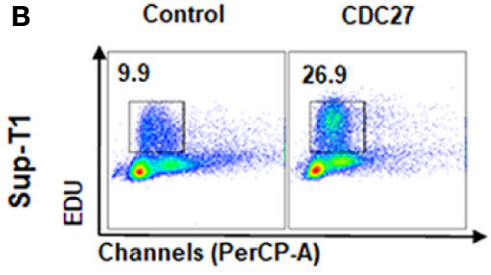

C

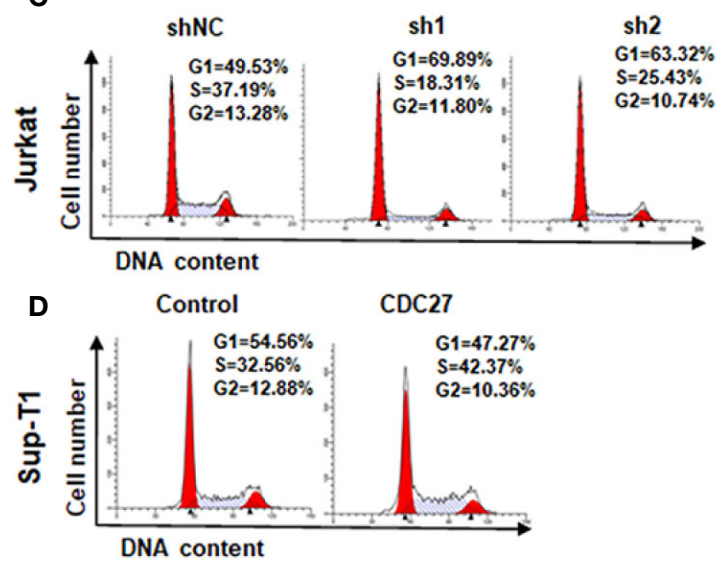

E

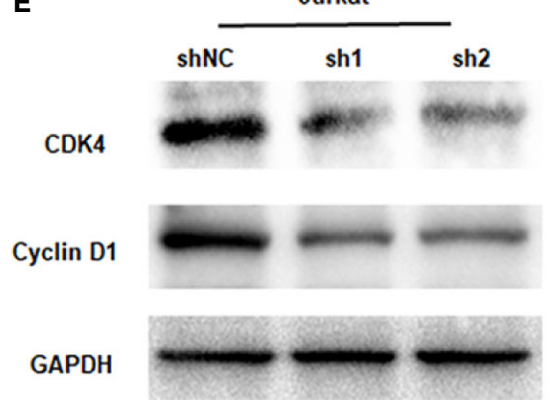

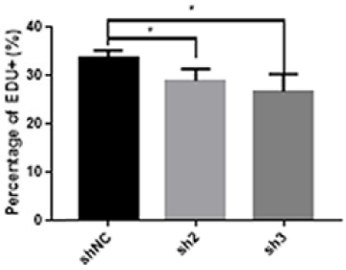
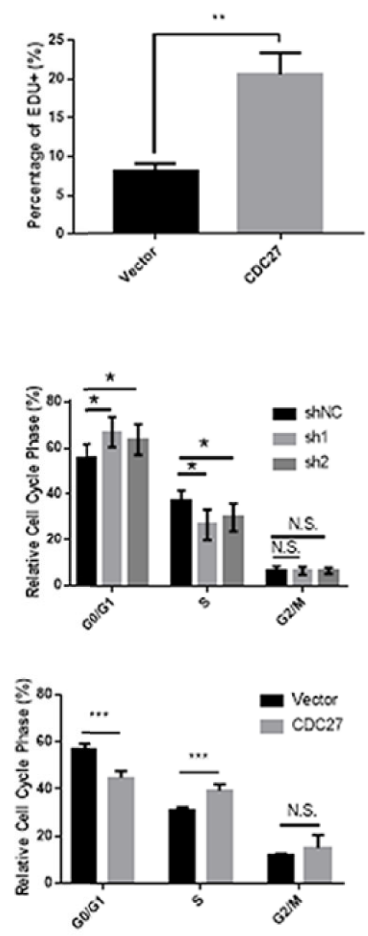

$\mathbf{F}$

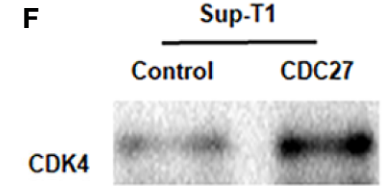

Cyclin D1

GAPDH

FIGURE 3 | CDC27 influence the G1/S phase transition. (A, B) Cell proliferation were assessed by EdU incorporation assay. Data are representative of at least three independent experiments. ${ }^{\star \star} P<0.01,{ }^{\star} P<0.05$. (C, D) Flow cytometry was used to examine the cell cycle by PI staining of both Jurkat and Sup-T1 cells. Images and qualification of the cell cycle distribution in three independent experiments are shown. ${ }^{\star} P<0.05,{ }^{\star \star \star} P<0.001$. N.S., not significant. (E, $\left.\mathbf{F}\right)$ Western blot was performed to detect the expression levels of cell cycle related proteins in both Jurkat and Sup-T1 cells, respectively. 

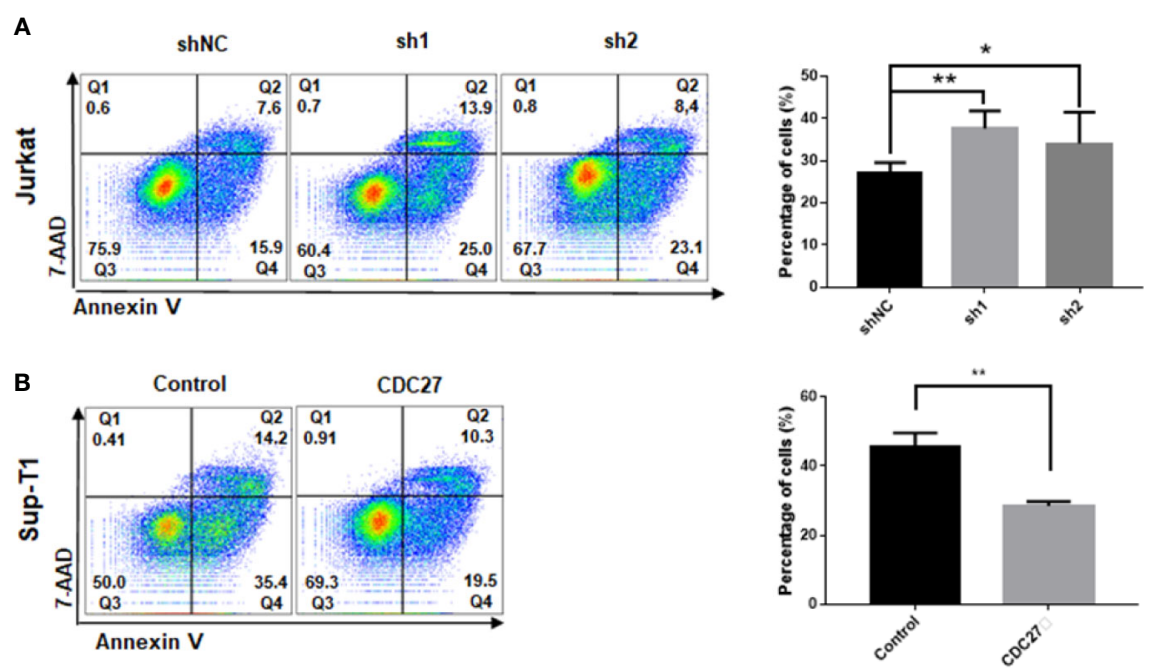

C

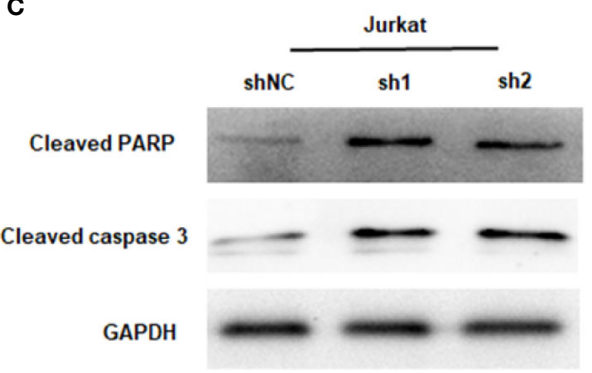

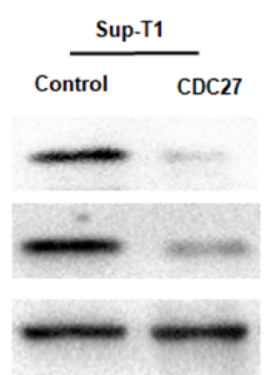

FIGURE 4 | CDC27 inhibits cell apoptosis in T-LBL cells. (A, B) Flow cytometry was used to examine the apoptosis as the sum of both Q2 and Q4 quadrants (early + late apoptosis) by Annexin V/7-AAD staining of both Jurkat and Sup-T1 cells. Apoptosis rates were expressed as the mean (Q2 + Q4) \pm SD of values from experiments performed in triplicate by using Student's t-test. ${ }^{*} P<0.05,{ }^{* \star} P<0.01$. (C, D) Western blot was performed to detect the expression levels of apoptotic related proteins in both Jurkat and Sup-T1 cells, respectively. 